\title{
Lo spazio carcerario come nonluogo di una soggettività negata ne Gli invisibili di Nanni Balestrini
}

\section{Vincenzo Binetti}

\section{(2) OpenEdition \\ Journals}

Edizione digitale

URL: http://journals.openedition.org/cei/275

DOI: $10.4000 /$ cei. 275

ISSN: 2260-779X

Editore

UGA Éditions/Université Grenoble Alpes

\section{Edizione cartacea}

Data di pubblicazione: 15 juin 2005

Paginazione: $75-87$

ISBN: 978-2-84310-066-6

ISSN: 1770-9571

Notizia bibliografica digitale

Vincenzo Binetti, «Lo spazio carcerario come nonluogo di una soggettività negata ne Gli invisibili di Nanni Balestrini», Cahiers d'études italiennes [Online], 3 | 2005, online dal 15 décembre 2006, consultato il 26 mars 2021. URL: http://journals.openedition.org/cei/275 ; DOI: https://doi.org/10.4000/cei.275 


\title{
LO SPAZIO CARCERARIO COME NONLUOGO DI UNA SOGGETTIVITÀ NEGATA NE GLI INVISIBILI DI NANNI BALESTRINI
}

\author{
Vincenzo Binetti \\ The University of Michigan
}

Quando la casa è in fiamme, si dimentica il pranzo.

- Sì : ma lo si va a riprendere nella cenere.

Nietzsche, Al di là del Bene e del Male.

\begin{abstract}
Abbiamo fatto i buchi in tutte le reti e poi abbiamo fatto le fiaccole le fiaccole si facevano con pezzi di lenzuoli legati stretti e poi imbevuti d'olio e allora anche lì all'ora stabilita nel mezzo della notte tutti accendevano l'olio delle fiaccole e infilavano questi fuochi nei buchi delle grate ma anche lì non c'era nessuno che li vedeva le fiaccole bruciavano a lungo doveva essere un bello spettacolo da fuori tutti quei fuochi tremolanti sul muro nero del carcere in mezzo a quella distesa sconfinata ma gli unici che potevano vedere la fiaccolata erano i pochi automobilisti che sfrecciavano piccoli lontanissimi sul nastro nero dell'autostrada a qualche chilometro dal carcere o forse un aeroplano che passa su in alto ma quelli volano altissimi lassù nel cielo nero silenzioso e non vedono niente ${ }^{1}$.
\end{abstract}

Così Nanni Balestrini ha voluto chiudere o forse lasciare provocatoriamente sospeso il percorso narrativo e geografico-esistenziale del suo protagonista nel tentativo di porre il lettore proprio a conclusione del romanzo davanti ad una possibile e necessariamente problematica presa di coscienza politica ed allo stesso tempo ad una "visualizzazione " di quello che

1. Nanni Balestrini, Gli invisibili, (prima edizione 1987), ora in La grande rivolta, Milano, Bompiani, 1999, propongo di aggiungere fra parentesi quadre : [infra Balestrini] a causa dei successivi rimandi alla stessa opera, p. 261. 
l'ideologia dello stato-nazione italiano cercava di relegare invece nella dimensione rassicurante dell'invisibilità, obliterando i desideri e i bisogni di una prorompente e destabilizzante soggettività collettiva nel luogo " in cui tutte le coazioni e le assurdità della società presente si condensano, il carcere $^{2} »$.

Ma è proprio ripercorrendo insieme all'io narrante quei luoghi della violenza e della coercizione che diventa possibile, almeno nella dimensione letteraria della vicenda narrata, superare il muro di cinta dello spazio carcerario ed entrare in contatto con un realtà sconvolgente e drammatica che possa però in qualche modo permettere una destabilizzante " porosità ", secondo l'accezione benjaminiana, tra il " dentro » e il " fuori ", tra spazio pubblico e privato, tra storia ufficiale e storie individuali e comunitarie.

Il romanzo si propone quindi come progetto ideologico-narrativo provocatorio rispetto all'orizzonte d'attesa di un pubblico di lettori abituato invece a filtrare la storia di quegli anni creativi e tumultuosi - e cioè gli anni delle lotte del movimento studentesco ed operaista, della scelta del rifiuto del lavoro e dell'omologazione, dell'autonomia, delle manifestazioni di piazza, delle occupazioni delle università e delle fabbriche - attraverso una più accomodante e mistificatoria rappresentazione mass-mediologica e culturale. Questo avviene nel romanzo anche e soprattutto tramite l'utilizzo di un referente documentaristico (l'intervista) e l'uso di un'oralità del linguaggio che tenta appunto di rendere più concreta e diretta la rappresentazione ; " oralità » intesa comunque non come semplice alternativa alla lingua scritta ma come traduzione/trascrizione sulla pagina di un discorso collettivo che era parte integrante della coralità e complicità emotiva e storico-politico-culturale di quegli anni ${ }^{3}$.

Le " lasse narrative " che definiscono l'unità ritmica del narrato e la divisione sistematica e strategica del testo secondo un " culto quasi fanatico del potere istitutivo del numero ${ }^{4} "$, - il testo si compone di 48 capitoli divisi a loro volta in paragrafi di circa 12-15 righe ciascuno - « un'os-

2. Rossana Rossanda, Storia crudele di Sergio l’invisibile, in "Il Manifesto ", 12 febbraio 1987, p. VII.

3. A tale proposito Italo Rosato sottolinea, prendendo come spunto la " lucida e nostalgicamente cattivante " prefazione di Calvino al Sentiero dei nidi di ragno, la differenza sostanziale che separa questa generazione da quella del periodo resistenziale : "I protagonisti del decennio trascorso hanno assistito alla dispersione delle loro utopie e - nei casi peggiori - assistono alla dissipazione delle loro esistenze individuali... Anche solo per la volontà di rompere il silenzio - ma certo non solo per questo - l'operazione di Balestrini [...] ci sembra di grandissima importanza. " (Italo Rosato, Nanni Balestrini. Gli Invisibili, in "Autografo ", n. 13, 1988, p. 116)

4. Giuliano Gramigna, Una lingua costruita per dire cose vedute e patite, in "Il corriere della sera ", mercoledì 28 gennaio 1987. 
sessione numerologica " come la definisce lo stesso Balestrini, danno quindi unità formale e stilistica al romanzo e fanno da contrappunto alla voluta mancanza di punteggiatura che indica ovviamente una scelta avanguardistica di rifiuto radicale della sintassi e del lessico tradizionale. Balestrini perciò pur conservando un rigore formale di fondo finisce, in effetti, col destabilizzare la struttura semantico-sintattica della lingua permettendo inoltre alla sua scrittura " parlata ", quasi " magnetofonica " di diventare strumento idoneo ed efficace al coinvolgimento emotivo e politico-intellettuale del lettore che proprio grazie ad accorgimenti stilistici precisi - la scorribilità e l'immediatezza del parlato, il tono conversativo della narrazione, le ripetizioni, l'uso costante dei deittici («questa cosa qui ", " un colpo proprio qui ", ecc.) - finisce potenzialmente col confrontarsi in maniera più diretta con la problematicità degli eventi narrati e con l'implicita estraneità dell'esperienza esistenziale e politica del protagonista e del movimento.

Un personaggio tra l'altro, questo Sergio a cui è dedicato il romanzo (si tratta di Sergio Bianchi, autonomo di Tradate, esule a Parigi), che per forza di cose diventa soggetto collettivo in quanto portavoce delle istanze comunitarie del gruppo, ma non per questo " rischia di fissarsi in uno stereotipo ${ }^{5}$ " e si propone invece come presenza corporea, intellettuale ed umana che pur nell'emblematicità e nella riconoscibilità immediata del suo essere soggetto narrante rappresentativo delle vicende degli anni '70 conserva una sua peculiare singolarità ed una sua contraddittoria identità : un’identità che come suggeriva, a suo tempo, Rossana Rossanda in una recensione al libro è " frutto d'una miniera, un pozzo di storia e idee sulla quale continua a regnare l'oscurità [e che] Balestrini... rende alla sua forza e fragilità ${ }^{6}$ ».

Ecco allora che già nell' incipit del romanzo siamo introdotti a questa " materialità del corpo sofferente, nudo, battuto, rotto, disteso, morto ${ }^{7}$ " attraverso la contestualizzazione del soggetto narrante, di questa voce che dice « io » ma sottende inevitabilmente la presenza comunitaria del « noi », all'interno dello spazio confinato ed alienante del carcere :

I sotterranei sono un dedalo di budelli illuminati ogni venti trenta metri da tubi al neon polverosi appesi a lunghi fili elettrici sbrindellati che pendono dal soffitto di cemento grezzo del sotterraneo spaccato da fenditure profonde lunghe... l'aria è umida e dalla bocca escono piccole nuvole di vapore quando si respira quell'aria nauseante... lo scalpiccio disordinato del piccolo corteo muto si mescola al tintinnare continuo delle catene rimbomba quando si

5. Lidia De Federicis, Il selvatico sul cemento, in "L'Indice dei libri del mese ", giugno 1987, n. 6, p. 8.

6. Rossanda, Storia crudele di Sergio l'invisibile, art. cit.

7. De Federicis, Il selvatico sul cemento, art. cit. 
attraversano le passerelle di legno fradicio... il piccolo corteo gira più volte a destra e a sinistra a sinistra e a destra fino a perdere del tutto l'orientamento ${ }^{8}$.

Ed è proprio già nella prima pagina che comincia a delinearsi anche un'interessante dinamica "spaziale " che caratterizzerà un po' tutto il romanzo e che vede appunto il protagonista muoversi secondo un percorso narrativo non lineare e orizzontale ma caratterizzato invece da continui flashback che sostengono in un certo senso la verticalità temporale di un problematico recupero memoriale di quegli eventi. Un percorso quindi nomadico e per certi versi contraddittorio ed imprevedibile che invita però il lettore a "re-imparare a pensare lo spazio ${ }^{9}$ " e rivisualizzare problematicamente sia i luoghi istituzionalizzati del potere, strumenti necessari attraverso cui lo stato-nazione impone inevitabilmente il suo bisogno " di disciplina e controllo ", per usare un'espressione foucaultiana, sia gli spazi deterritorializzati liberatori e creativi della strada, delle aree occupate e della rivolta dove "l'energia [...] è più disordinata e ha meno orizzonti della pulsione politica : è più scatenata, si dissipa di più ${ }^{10}$ ".

Nella continua tensione dinamica tra questi due momenti si delinea così il progetto autoriale che, attraverso l'utilizzo di specifiche analogie, metafore e flashback riesce appunto a dare funzionalità alle proprie intenzioni ideologiche ed alle proprie strategie linguistico-formali. Ecco allora come, ad esempio, la rappresentazione dello spazio della scuola o della fabbrica ${ }^{11}$ richiami emblematicamente quella immediatamente precedente del luogo carcerario sia nella descrizione e nella posizionalità degli oggetti - cancelli/sbarre di ferro/corridoi/muri di cemento - che nelle dinamiche conflittuali del soggetto narrante con i detentori del potere, professori/guardie carcerarie/padroni :

È arrivato il giorno stabilito e la mattina presto prima che aprono i cancelli avevamo attaccato un grande manifesto che annunciava l'assemblea e invitava tutti a partecipare... il preside Mastino arriva come il solito per primo e si mette a leggere il manifesto poi gonfia la

8. Balestrini, op. cit., p. 93.

9. Marc Augé, Nonluoghi. Introduzione a una antropologia della surmodernità, Milano, Elèuthera, 2002, p. 37. (Titolo originale : Non-lieux. Introduction à une anthropologie de l'espace, Paris, Seuil, coll. «Librairie du xxe siècle », 1992).

10. Jean-Luc Nancy, La città lontana, Verona, Ombre corte, 2002, p. 51. (Titolo originale : Los Angelès ou la ville au loin, Paris, Mille et une nuits, 1999).

11. In questi termini infatti l'autore descrive più avanti la condizione di alienazione e di sfruttamento all'interno della fabbrica : « io non sapevo bene cos'era quella fabbrica la vedevo dall'esterno come una cosa mostruosa e sporca che scaricava fumi nell'aria e liquidi puzzolenti nel fiume che gli scorre di fianco l'impressione che ho avuto la prima mattina di lavoro è stata dura [...] mi hanno indicato dove dovevo andare e già lì subito mi è venuto voglia di andarmene via di girare le spalle e via uscire di lì e andarmene quando ho visto il mio reparto una specie di corridoio lungo e stretto senza finestre c'erano solo dei grandi lucernai in alto e una puzza tremenda di solventi [...] gli operai avevano tutti dei grembiuli neri tranne il caporeparto che aveva un grembiule bianco e che stava nel suo ufficio in fondo al corridoio chiuso da una vetrata da dove poteva vedere controllare tutto il reparto. " (Balestrini, op. cit., p. 203) 
mascella e ci guarda da cattivo... poi arrivano i professori che leggono e non commentano si limitano a guardarci come dei pazzi dopo qualche minuto esce fuori una schiera di bidelli che Mastino gli aveva dato l'ordine di strappare via i manifesti $[\ldots]$ mi sembrava di vedere il padrone che passeggia davanti alla fabbrica in quelle storie che avevo letto sulle prime lotte operaie sui primi scioperi lo stesso metodo di intimidazione ${ }^{12}$

Gli spazi interni del carcere, come molto significativamente in parallelo anche quelli esterni delle istituzioni e della fabbrica diventano perciò dei "nonluoghi " all'interno dei quali non si creano " né identità singola, né relazione, ma solitudine e similitudine... essi non operano alcuna sintesi, non integrano nulla, autorizzano solo [...] la coesistenza di individualità distinte simili e indifferenti le une alle altre ${ }^{13}$ ». Ecco perché le mobilitazioni di piazza "fuori » e la rivolta del carcere "dentro " rappresentano dei segnali emblematici del bisogno di rimappare seppur momentaneamente lo spazio, di deterritorializzare questi nonluoghi per tentare di immaginare una vivibilità diversa del proprio corpo attraverso la temporanea definizione di aree di autonomia e di creatività che si sottraggano alle imposizioni autoritarie del potere costituito :

In città i circoli giovanili hanno organizzato una festa in piazza del duomo io e China ci andiamo in treno da soli arriviamo in anticipo all'appuntamento con gli altri nostri compagni c'è già moltissima gente la polizia presidia in forze tutto intorno si fanno scritte sui muri e per terra lo spazio è un diritto oppure per la società della festa oppure riprendiamoci la vita [...] proviamo a metterci in cordoni e viene fuori un serpentone mica male vediamo gli altri del nostro collettivo sono venuti tutti stanno a gruppetti mischiati con gli altri la testa del corteo parte decisa in direzione della piazza del duomo ${ }^{14}$

La guerriglia urbana destabilizza e sconvolge temporaneamente dunque l'immobilità dello spazio " rigido " della città secondo una planimetria rizomatica che non è più quella pianificata ed omologante della cartina turistica, emblematicamente rappresentata dal riferimento alla piazza Duomo, ma diventa quella metamorfica e costituente dell'azione (rivoluzione) dirompente e creativa del movimento. Le strade, le piazze e i luoghi simbolici della storia ufficiale della metropoli si trasformano così in aree " di passaggio ", in zone indefinibili di imprevedibilità che permettono un'auspicabile riterritorializzazione dello spazio urbano ed all'interno delle quali diventa possibile operare nomadicamente in maniera fluida e dinamica ${ }^{15}$, ma soprattutto "festosa ", proprio perché come suggerisce Lefebvre i "festivals " rappresentano "Dionysiac life

12. Balestrini, op. cit., p. 96.

13. Augé, op. cit., p. 95 e 101.

14. Balestrini, op. cit., p. 99.

15. Scrive Lefebvre : «Representational space... may be directional, situational or relational, because it is essentially qualitative, fluid and dynamic. " Henri Lefebvre, The Production of Space, Oxford, Basil Blackwell, 1991, p. 42. (Titolo originale : La production de l'espace, Paris, Anthropos, 1986, rist. 2000). 
[and] differed from everyday life only in the explosion of forces which had been slowly accumulated in and via everydaylife itself [...] revolutions of the past were festivals ${ }^{16}$ ".

Ma è proprio in netta opposizione a questa rappresentazione di una spazialità festosa che l'autore trasferisce nuovamente, nelle pagine successive (in modo volutamente provocatorio e traumatico per il lettore) azione e descrizione all'interno del carcere. La storia del protagonista ritorna così - grazie all'utilizzo costante del flashback, che permette appunto una frammentarietà continua della linearità spaziotemporale del narrato - nel « nonluogo ${ }^{17}$ " carcerario dove la volontà di controllo e di coercizione dell'autorità si estrinseca simbolicamente ed in maniera macroscopica proprio nella organizzazione di una struttura rigida e strategicamente pianificata all'interno della quale i singoli individui sono obbligati a muoversi meccanicamente e senza senso seguendo percorsi predefiniti e pilotati :

Sono arrivato in cella e erano passati pochissimi minuti da quando ero tornato in cella quando ho sentito delle urla provenire dalla rotonda devo spiegare cos'è la rotonda la sezione speciale del carcere dove eravamo era una palazzina a pianta rettangolare una palazzina di tre piani pianterreno primo piano e secondo piano e ogni piano era diviso in due bracci al centro di questi due bracci a tutti i piani c'erano due cancelli e in mezzo tra i due cancelli c'era uno spazio che era la rotonda appunto la rotonda dove arrivavano le scale e di lì si smistava la gente in un braccio o nell'altro braccio il braccio destro da una parte e il braccio sinistro dall'altra io ero al braccio sinistro dell'ultimo piano cioè del secondo piano ${ }^{18}$

Diventa perciò a mio avviso sintomatico dell'intenzione ideologica autoriale e del complessivo evolversi della vicenda narrata questa progressione spaziale continua e parallela tra luogo e comunità politica che vede appunto il momento creativo ed antagonistico del movimento occupare uno spazio operativo che per forza di cose tende ad espandersi e a ridefinire continuamente i suoi confini, mentre, in maniera diametralmente opposta, alla conseguente repressione da parte dello Stato, ma anche in un certo senso all'intrinseco " svanire ${ }^{19}$ " del movimento e dei

16. Lefebvre, Critique of Everyday Life, citato in Andy Merrifield, Metromarxism. A Marxist Tale of the City, New York and London, Routledge, 2002, p. 83.

17. Secondo Marc Augé « La surmodernità trova naturalmente la sua espressione completa nei nonluoghi $[\ldots]$ nei nonluoghi vi è sempre un posto specifico per delle curiosità presentate come tali ». Ecco allora perché, continua Augé, a differenza della modernità, "lo spazio della surmodernità è invece segnato da questa contraddizione : esso ha a che fare solo con individui ma questi sono identificati, socializzati e localizzati solo all'entrata o all'uscita ". (Augé, op. cit., p. 99-101).

18. Balestrini, op. cit., p. 110.

19. Scrive a questo proposito Balestrini : "Si cercava di dormire nelle case di quei compagni che si ritenevano meno conosciuti meno esposti o meglio ancora nelle case di amici che non c'entravano niente o nelle case di amici di amici le manifestazioni e le feste di piazza erano finite da un pezzo il movimento era come un fantasma assente ripiegato su se stesso rintanato nei suoi ghetti la scena adesso era occupata dallo stillicidio di azioni armate clandestine rivendicate da decine di sigle di organizzazioni 
suoi desideri e velleità, fa da controparte un inevitabile restringersi ed " irrigidirsi » di quello stesso spazio.

Ed in effetti le pulsioni creative e rivoluzionarie della "strada " vengono ostacolate e contenute dalla polizia e dagli organi di controllo dello stato proprio perché, come ci ricorda Debord, « l'effort de tous les pouvoirs établis [...] pour accroître les moyens de maintenir l'ordre dans la rue, culmine finalement dans la suppression de la rue ${ }^{20}$ ", e questo lo si vede nella rappresentazione atroce e fortemente "realistica » della morte dello studente investito dal " jeeppone » della polizia (per la cronaca, si tratta di Giannino Zibecchi, ucciso a Milano il 17 aprile del 1975) :

Urla fortissime grida vedo molti compagni che corrono verso quel punto non riesco a vedere niente c'è fumo e confusione tutti hanno gli occhi rossi che piangono per i lacrimogeni scendo dalla saracinesca e vado verso quel punto correndo insieme a altri ci scontriamo con altri che vengono in senso opposto facce disperate occhi sbarrati alcuni abbassano i fazzoletti uno si mette le mani nei capelli non riesco a vedere cosa è successo c'è un gruppo di compagni fermi a semicerchio alcuni piangono non è per i lacrimogeni alcuni singhiozzano una ragazza grida qualcosa che non capisco poi più in là vedo il corpo insanguinato per terra vedo la lunga scia di sangue scuro e più in là vedo la massa rossiccia del cervello che la ruota del jeeppone gli ha schizzato fuori dalla testa schiacciata ${ }^{21}$

Oltre ad essere un reperto documentaristico della storia e della memoria collettiva di quegli anni questo episodio di cronaca si inserisce nella dimensione " fittizia " del narrato visualizzando davanti agli occhi del lettore un'immagine tanto riconoscibile ed indimenticabile per la sua assurdità quanto sconvolgente per le sue implicazioni etico-politiche ; ma essa serve anche ad anticipare analogicamente quello che succederà nel carcere con la rivolta e la violenza inaudita della sua repressione. Ed è così che, parallelamente, pur nel riconoscimento autoriale dell'impossibilità di " raccontare 22 " la totalità storico-oggettiva di queste vicende, si dipana secondo il ritmo incalzante dell'oralità anche il tentativo di resistenza e di autonomia dei prigionieri all'interno del carcere :

combattenti che si facevano concorrenza la vita del movimento era finita ma per i compagni non era finita non è che potevano mettersi da parte e dire aspettiamo stiamo a vedere perché per la repressione tutti erano coinvolti non si facevano troppe distinzioni. " (Balestrini, op. cit., p. 104).

20. Guy Debord, La société du spectacle, Paris, Gallimard, coll. Folio, 1992 [1967], p. 166 [\$ 172].

21. Balestrini, op. cit., p. 101-102.

22. Scrive Balestrini : "E allora a questo punto non mi ricordo più dove ero rimasto in tutta questa storia anche perché ci sono un sacco di cose che non mi ricordo che non mi ricordo più bene precisamente come sono successe e ci sono anche un sacco di cose che non si possono ricordare che si possono solo dimenticare non è che qui io voglio raccontare tutta la storia della mia vita e neanche voglio raccontare tutto quello che è successo in questo periodo in cui sono successe tante cose diverse di tutti i tipi contraddittorie che metterle tutte insieme cercare di dargli un senso mi sembra proprio impossibile ma quello che mi interessa qui adesso è soltanto raccontare ma così dal mio punto di vista naturalmente queste storie che mi sono successe così perché forse adesso vale la pena di raccontarle. " (Balestrini, op. cit., p. 181). 


\section{VinCENZO BinetTi}

Quello che è successo e che si è saputo poi dopo o almeno in parte perché non è che poi tutto si può sempre raccontare di queste storie è stato che velocissimamente i compagni che avevano sequestrato le guardie sono scesi giù con le chiavi che avevano preso alle guardie hanno aperto il cancello... io a questo punto ho visto della gente mascherata che sono arrivati nel mio braccio sono arrivati davanti alla mia cella e hanno aperto tutte le celle del braccio sinistro hanno aperto anche la mia cella e allora c'era una grande confusione... e poi tutti quanti ci siamo riversati fuori nel corridoio... e da quel momento ha preso avvio la rivolta (ibid., p. 112)

La percorribilità del luogo carcerario viene perciò ridefinita e riterritorializzata permettendo degli spostamenti e delle interazioni tra gli individui che, almeno nella cosciente transitorietà dell'evento, prima cioè della sua " necessaria " e violenta repressione ${ }^{23}$, possono esprimere comportamenti non codificati, ma spontanei e liberatori ; questo determina, anche all'interno di uno spazio sinonimo di alienazione ed angoscia, una momentanea situazione di euforia e di festa :

Il clima che c'era era di euforia c'era un clima di festa io mi ricordo di questa enorme euforia di questa eccitazione di questa festa e quello che tutti dicevano in continuazione e di cui eravamo convinti era che mai e poi mai ci poteva essere un intervento militare da parte delle guardie dei carabinieri della polizia... mi ricordo che non c'erano preoccupazioni mi ricordo che non c'era assolutamente nessuna ansietà mi ricordo che c'era euforia e eccitazione c'era questo meccanismo che era scattato nella testa di tutti di considerare questa situazione come non pericolosa e che la faceva vivere a tutti come una festa ${ }^{24}$

L'efficace strategia autoriale permette al lettore di visualizzare quindi l'impossibile, di attraversare cioè la dimensione " altra " dell'esperienza carceraria e di confrontarsi, forse anche involontariamente - proprio perché « vi sono spazi in cui l'individuo si mette alla prova come spettatore senza che la natura dello spettacolo lo interessi veramente ${ }^{25}$ " - con le ragioni della rivolta. Questo determina inoltre una problematica presa di coscienza delle forme di resistenza e di lotta operanti all'interno del carcere che pur nell'implicito riconoscimento della loro transitorietà ${ }^{26}$

23. L'immagine agghiacciante della repressione all'interno del carcere è resa emblematicamente attraverso la rappresentazione " realistica " di un corpo ancora una volta martoriato, offeso e violato : " picchiavano con manganelli con bastoni con spranghe di ferro e questo compagno che era piccolo lo hanno proprio massacrato di botte poi un'altra scena che ho visto è stato uno che l'hanno preso per i capelli dopo averlo pestato a terra l'hanno tirato su per i capelli e l'hanno messo contro il muro e poi uno con una spranga di ferro gliel'ha data sulla faccia proprio così un colpo con la spranga di ferro così di traverso sulla faccia e gli hanno spaccato il naso e la fronte [...] queste erano le cose che si vedevano mentre stavamo lì al buio dietro la rete metallica impotenti " (Balestrini, op. cit., p. 170). Mi sembra importante sottolineare a questo proposito come lo «spettacolo " del massacro riproponga anche qui una dinamica tra il dentro e il fuori : "fuori " cioè si trova anche il lettore, estraneo alla vicenda, ma che visualizza impotente la scena, come impotenti sono i prigionieri di fronte alla violenza « legalizzata » della polizia e dello Stato.

24. Balestrini, op. cit., p. 124.

25. Augé, op. cit., p. 80.

26. E'sintomatico ciò che afferma a questo proposito il protagonista : « [...] io penso e anche tanti come me lo pensano che in fondo non abbiamo mai avuto non solo non abbiamo mai avuto nessuna idea né voglia di vincere ma nemmeno nessuna idea che c'era qualcosa da vincere da qualche parte e poi 
testimoniano tuttavia le ragioni politiche di una protesta, e rendono soprattutto credibile la possibilità di reinventare e rimappare costantemente, in maniera appunto anche festosa ed euforica, i nonluoghi dell'alienazione e del sopruso.

Ma ancora una volta il susseguirsi strategico delle sequenze narrative permette una permeabilità tra il " dentro " e il "fuori ", un continuo e parallelo confrontarsi dialettico della sfera pubblica e di quella privata ${ }^{27}$. Loccupazione del Cantinone da parte del movimento rappresenta infatti un'immagine concreta e non utopica della possibilità reale di « evadere ", anche in termini di struttura narrativa, dalle costrizioni dello spazio carcerario, e riproporre anche nel luogo "pubblico » situazioni creative di autonomia. L'appropriazione di uno spazio semiabbandonato e decrepito come il Cantinone mette in moto perciò non solo un processo di trasformazione e di produttività economico-politica e culturale autogestita, ma permette anche e soprattutto una stimolante contaminazione tra il movimento e diverse realtà comunitarie urbane (disoccupati, lavoratori, artisti, anziani, bambini, ecc.) ${ }^{28}$ proprio perché, come suggerisce Berman, " their initiatives showed that obscure and decaying old places could turn out to be - or could be turned into - remarkable public spaces ${ }^{29}$ \%. La caoticità creativa che contraddistingue quindi l'energia operante delle varie comunità che si muovono attraverso questo spazio, e cioè dentro e fuori dell'area occupata, non può che essere ancora una volta festosa ed euforica :

La festa era al massimo c'era una grande euforia c'era una grande eccitazione gente che entrava e che usciva in continuazione una confusione indescrivibile tutti erano entusiasti di quel posto dicevano che dovevamo restarci che dovevamo restare lì a tutti i costi che avremmo fatto delle cose fantastiche nel Cantinone la musica suonava al massimo... tutti guardavano il palco dove uno adesso cantava mi piace molto di suonare e con la musica pestare ma non mi guadagno il pane perché suono come un cane sono un teppista c'ho in testa la conquista

sai se ci penso bene adesso a me la parola vincere mi sembra proprio uguale come a morire. » (Balestrini, op. cit., p. 131)

27. Non è un caso infatti che la storia con China cominci proprio durante l'occupazione del Cantinone : "China la prima volta che l'ho conosciuta è stato quando c'è stata l'occupazione del Cantinone e lì che l'ho vista per la prima volta China era arrivata lì non so quando e stava aiutando Gelso a fare il murales che Gelso aveva deciso di fare sulla parete più grande aveva un grande pennello e lo intingeva in un secchio di tempera bianca ma lo intingeva troppo e la pittura schizzava dappertutto e colava giù sul pavimento io ho visto quel disastro e sono andato lì per farle vedere come doveva fare ma anche perché mi sembrava molto carina. " (Balestrini, op. cit., p. 117).

28. Scrive infatti Balestrini : " intanto altra gente cominciava a arrivare arrivavano a gruppi gli studenti che già sapevano della cosa e poi arrivavano i primi curiosi arrivavano operai e disoccupati che avevano visto i nostri manifesti e i volantini si era sparsa la voce e la gente arrivava entrava e si aggirava per lo stabile guardando dappertutto noi spiegavamo perché abbiamo occupato cosa vogliamo fare adesso e la gente discuteva domandava [...] c'erano i bambini che correvano su e giù per il salone salivano nelle stanze sopra c'era dappertutto un caos completo." (Balestrini, op. cit., p. 119)

29. Marshall Berman, All That is Solid Melts into Air, New York, Simon and Schuster, 1982, p. 321. 
sono un po' brutale ma ti giuro son normale e io sono andato a mettermi con China proprio sotto il palco mentre la musica suonava al massimo 30

Queste situazioni di aggregazione spontanea che si delineano nella transitorietà nomadica degli spazi di autonomia sono, come si è detto, per forza di cose momentanee e " costituenti ", ma rappresentano comunque un segnale emblematico della condizione di crisi e di disagio di un'intera cultura e quindi denunciano la necessità di un bisogno radicale di cambiamento. Non è un caso, infatti, che la dimensione alienante che caratterizza il rapporto conflittuale del soggetto narrante con il suo spazio si riproponga anche nel luogo soffocante e retrogrado del paesino natale :

Quello dove io vivevo era un paese di merda e anche la gente era gente di merda non mi piaceva questo paese e non mi piaceva questa gente [...] questi paesi se non li conosci se non li abiti ti puoi confondere puoi davvero scambiare un paese con l'altro sono tutti uguali al centro c’è la piazza che è poi sempre la piazza della chiesa e tutti hanno sempre la stessa strada principale che passa in mezzo al paese con qualche negozio e i bar la scuola e il municipio [...] e la strada principale che attraversa la piazza e da una parte porta al cimitero e dall'altra alla stazioncina della ferrovia che collega tutti quanti i paesini tra di loro (Ibid., p. 154)

Qui sembrano riproporsi, appunto, in maniera allucinante le stesse costrizioni del nonluogo carcerario attraverso una dinamica narrativa che fa parte comunque di un più ampio discorso letterario-culturale riguardante il rapporto problematico città-campagna e che serve in un certo senso ad allargare il discorso portato avanti in questo romanzo in una dimensione più complessa che va ben oltre il referente testuale e l'evolversi della vicenda narrata, ma riguarda anche questioni ed istanze metatestuali e metastoriche di più ampio respiro. In questo senso ha forse ragione Guglielmi, anche se a mio avviso non si può prescindere comunque dalla referenzialità storico-politica della trama, quando sostiene che nel romanzo « la disperazione politica diventa disperazione esistenziale ${ }^{31}$ ".

Mi sembra tuttavia non casuale che verso la fine del romanzo, quasi a sancire l'estinguersi della parentesi creativa e dirompente degli anni '70 e del movimento, e quasi a sottolineare con più forza il fallimento implicito dell'intenzionalità " educativa " dell'istituzione carceraria, l'autore abbia voluto rappresentare emblematicamente il suicidio di uno dei protagonisti di questa storia non dentro ma fuori dal carcere, attraverso un'identificazione allucinante ma significativa della cella con la stanza della casa di famiglia. Una presa di coscienza, insomma, filosofico-esistenziale e non solo politica del fatto che la condizione di solitudine e di alienazione non riguarda unicamente la " mostruosità " per certi versi

30. Balestrini, op. cit., p. 126.

31. Guido Guglielmi, Nanni Balestrini. Gli Invisibili, in «Il Verri », n. 7, settembre 1988, p. 138. 
rassicurante, perché confinata, di quello spazio ma si ripropone ed amplifica problematicamente anche « altrove » :

Gelso sembrava ormai non riconoscere più nessuno [...] aveva chiesto ai suoi genitori di non fare entrare nessuno nella sua camera e anche lui non usciva mai dalla sua camera si faceva anche portare da mangiare nella sua camera e in pochi giorni ha trasformato la camera in una cella $[\ldots]$ e ha cominciato anche a arredarla come una cella con quelle cose che usano i detenuti $[. .$.$] e poi una sera ha mimato un'evasione ha annodato le lenzuola e si è calato$ dalla finestra l'hanno trovato nel cortile con una caviglia slogata [...] ha passato un mese senza mai uscire dalla sua camera viveva come in prigione [...] e dopo un mese un giorno l'hanno trovato impiccato nella sua cella che era la sua camera una mattina l'hanno trovato lì che si era impiccato con le lenzuola annodate con cui aveva mimato l'evasione a cui aveva sempre pensato e che neanche adesso gli era riuscita ${ }^{32}$

La " fuga » necessaria da questi nonluoghi di alienazione omologante "spécialement organisés pour les fins de cette pseudo-collectivité qui accompagne aussi l'individu isolé dans la cellule familiale ${ }^{33}$ " non si risolve comunque attraverso un prevedibile rifugiarsi romantico in un "fuori " inesistente e perciò utopico, ma trova invece possibilità pragmatiche di realizzazione concreta proprio nella continua e contingente operosità politica del movimento ${ }^{34}$, che nel suo procedere nomadico attraverso gli spazi pubblici della modernità tenta continuamente di immaginare e re-inventare possibili aree di sopravvivenza e di autonomia, "where people dynamically and spontaneously interact with their surroundings, surroundings where the antithesis between our inner and outer worlds [...] has been collapsed ${ }^{35} \%$.

Non penso dunque che si possa parlare, a proposito di questo romanzo, come vuole Spinazzola, di " una sorta di elogio funebre, in forma romanzesca, del movimento dell'Autonomia [...] una vocazione alla disfatta che è in definitiva un abbandono all'istinto di morte ${ }^{36}$ » oppure, come sostiene Colombo, " di desolazione senza ritorno ${ }^{37}$ ", proprio perché in fin dei conti la potenzialità rivoluzionaria di questo processo di

32. Balestrini, op. cit., p. $259-260$.

33. Debord, op. cit., p. $166(\$ 172)$.

34. Ecco perché il movimento assume per il protagonista i contorni di una grande famiglia : « il mio ruolo è quello di uno che sta andando in galera adesso pensavo ai compagni e questo mi consolava perché pensavo che adesso tutti si stavano mobilitando si stavano dando da fare per me non mi avrebbero lasciato solo e ero orgoglioso del fatto che avevo tutti questi compagni questa grande famiglia che si prendeva a carico la mia situazione i miei problemi che avrebbero pensato a tutto [...] sentivo che non ero solo facevo parte di una forza collettiva e questo mi dava una grande forza [...]» (Balestrini, op. cit., p. 179).

35. Merrifield, Metromarxism. A Marxist Tale of the City, New York and London, Routledge, 2002, p. 181.

36. Vittorio Spinazzola, Requiem per gli Invisibili, in «L'Unità », 22 febbraio 1987.

37. Furio Colombo, Voci di dolore sulle rovine del terrorismo, in "Tuttolibri » (La Stampa »), 4 aprile 1987. 
destabilizzazione dell'apparato politico-economico su cui si regge la logica autoritaria dello stato-nazione e che trova appunto nella condizione alienante del carcere, della fabbrica e della scuola una sua emblematica rappresentazione, testimonia invece, come suggerisce lo stesso protagonista attraverso un riferimento testuale che è anche e soprattutto metafora meta-narrativa della posizionalità ideologica autoriale, la volontà ferrea e fortemente politica "dell'importanza di scrivere ${ }^{38}$ ".

$\mathrm{Ed}$ anche se è proprio lo stesso Balestrini a sostenere che in fondo " la letteratura non serve sul piano immediato della prassi 39 ", in questo romanzo la scrittura diventa tuttavia testimonianza esemplare di una «storia » collettiva e personale, strumento idoneo di una voluta partecipazione politica ad un processo di " riscrittura " delle vicende di quegli anni e quindi del bisogno implicito e necessario di coinvolgimento e contaminazione intellettuale ed emotiva da parte del pubblico di lettori forse perché " è [proprio] nell'anonimato del nonluogo che si prova in solitudine la comunanza dei destini umani ${ }^{40}$ ».

Il problema fondamentale è allora quello di sforzarsi in qualche modo di rendere perlomeno "intuibile " l'invisibilità di questa esperienza letteraria e storico-politica e trasformare magari quel nonluogo cimiteriale che è appunto la prigione dopo la repressione della rivolta, ma che è anche qualsiasi strada o piazza dopo la fine della « festività » rivoluzionaria degli anni ' 70 , in un qualcosa di nuovamente vivo e pulsante. Un invito insomma ad operare un atto di resistenza e di protesta nei confronti di una società "sorda " e " cieca " mettendo a fuoco davanti ai nostri occhi " almeno una volta " questa realtà fortemente problematica, provando a liberarci da certi " automatismi verbali e mentali ${ }^{41}$ ", e a riascoltare quelle " voci » e visualizzare quei corpi e quelle presenze per tentare così di dar spazio alle loro storie mentre gli altri " volano altissimi lassù nel cielo nero e silenzioso e non vedono niente ${ }^{42} »$.

38. Balestrini, op. cit., p. 207.

39. N. Balestrini, Prendiamoci tutto : conferenza per un romanzo, Milano, Feltrinelli, 1972, p. 16.

40. Augé, op. cit., p. 110.

41. Rosato, art. cit., p. 121.

42. Balestrini, Gli invisibili, op. cit., p. 261. 


\section{Opere citate}

Augé Marc, Nonluoghi. Introduzione a una antropologia della surmodernità, Milano, Elèuthera, 2002.

Balestrini Nanni, Gli invisibili, (prima edizione 1987), ora in La grande rivolta, Milano, Bompiani, 1999.

-, Prendiamoci tutto : conferenza per un romanzo, Milano, Feltrinelli, 1972.

Berman Marshall, All That is Solid Melts into Air, New York, Simon and Schuster, 1982.

Colombo Furio, Voci di dolore sulle rovine del terrorismo, in "Tuttolibri » («La Stampa »), 4 aprile 1987.

De Federicis Lidia, Il selvatico sul cemento, in "L'Indice dei libri del mese ", giugno 1987, n. 6.

Debord Guy, La société du spectacle, Paris, Gallimard, coll. Folio, 1992 (1967).

Esposito Roberto, Le ideologie della neoavanguardia. Napoli, Liguori, 1976.

Gemelli M. e Piemontese, F. (a cura di), L'invenzione della realtà. Conversazione su letteratura e altro. Napoli, A. Guida Editore, 1994.

Guglielmi Guido, Nanni Balestrini. Gli Invisibili, in « Il Verri », n. 7, settembre 1988.

Gramigna Giuliano, Una lingua costruita per dire cose vedute e patite, in "Corriere della sera ", mercoledì 28 gennaio 1987.

Lefebvre Henri, The Production of Space, Oxford, Basil Blackwell, 1991.

Marcoaldi Franco, L'Autonomia è un romanzo. Colloquio con Nanni Balestrini, in "L'Espresso », 18 gennaio, 1987.

Merrifield Andy, Metromarxism. A Marxist Tale of the City, New York and London, Routledge, 2002.

Nancy Jean-Luc, La città lontana, Verona, Ombre corte, 2002.

Pampaloni Geno, La solida prosa di un eversore, in "Il Giornale », 21 febbraio 1987.

Renello Gian Paolo, I labirinti di Balestrini, in " Il Verri », settembre-dicembre 1993.

Rossanda Rossana, Storia crudele di Sergio l'invisibile, in "Il Manifesto ", 12 febbraio 1987.

Rosato Italo, Nanni Balestrini, Gli Invisibili, in "Autografo », n. 13, 1988.

Sanguineti Edoardo, Come agisce Balestrini, in "Il Verri », vol. 7, 1963.

Spinazzola Vittorio, Requiem per gli Invisibili, in "L'Unità », 22 febbraio 1987.

Tadiotto Antonio, L'orda invisibile di Nanni Balestrini, in N. Roelens e I. Lanslots (a cura di), Piccole finzioni senza importanza. Valori della narrativa italiana contemporanea. Ravenna, Longo, 1993. 